\title{
Effect of cue salience on discrimination learning
}

\author{
STUART I. OFFENBACH \\ Purdue University, West Lafayette, Indiana 47907
}

\begin{abstract}
Salience for specific dimensional cues was assessed in second-grade children immediately prior to a two-choice discrimination learning task. The cue reinforced in the learning task was either the salient cue (learned fastest), the alternate cue of the salient cue's dimension, or a cue from a dimension not salient (learned slowest). Children without specifically salient cues learned in approximately as many trials as did the children who were reinforced for choices of the salient cue or dimension. The results indicated that specific cue salience did not have a general facilitative effect, but that it was related to discrimination learning in a logical and predictable manner.
\end{abstract}

The role of dimensional salience, dominance, or preference in discrimination learning continues to be of interest in the study of development (cf. Esposito, 1975). Dimensional salience is presumed to facilitate learning when the salient dimension is relevant or to retard learning when the salient dimension is irrelevant. However, there are issues in this area that have not been directly considered. First, the procedure normally used to determine salience is based on dimensional responding rather than choices of specific cues. The subject normally matches one of two sample stimuli to a standard (each sample shares one cue or dimension with the standard). For example, in a color-form task, the choice of the comparison stimulus that is the same color as the standard of a matching-to-sample task implies that all color cues will be more salient than the shape cues. However, while red may be more salient than blue, square, or circle, it may also be the case that square is more salient than blue or circle. In a study related to this issue, Offenbach, Baecher, and White (1973) reported that less than half their Grade 3 subjects chose a single cue in a "cue salience" task from the same dimension they used in a "dimensional salience" task. Thus, rather than always examining the role of dimensional salience in learning, an alternative strategy would be to determine which single cue is salient and then to try to find out how discrimination task acquisition is related to that cue (rather than always looking at dimensional salience). A related issue in salience studies concerns the task. Performance in a shift or transfer task is the focus of the analyses rather than initial learning. Since relative salience is affected by learning (Tighe, Tighe, Waterhouse, \& Vasta, 1970), the potential interaction between salience and the change in reinforcement contingencies at the time of the shift may distort the

The author thanks Mr. Royce Kurtz of the Tippecanoe School Corporation for providing a source of subjects for this research. Reprint requests should be sent to Stuart I. Offenbach Department of Psychological Sciences, Purdue University, West Lafayette, Indiana 47907. effects of salience. In support of this possibility, Cole (1976) reported that response strategies used in transfer often are different from those used in initial learning tasks.

\section{METHOD}

The purpose of this study was to examine the effects of specific cue salience on the acquisition of a discrimination learning task. A cue salience task was presented to 111 children aged 7 and 8 years (Grade 2). The task consisted of one presentation each of the eight stimulus pairs formed by combining the binaryvalued dimensions of color (red and blue), shape (square and circle), size (large and small), and position (left and right) (e.g., large blue circle on left, small red square on right). The children were asked to point to one stimulus of each pair. After the response, the next pair was presented and the child chose again. No feedback was given, but the children were told after the eighth trial that they had done well. A cue selected on seven or eight of the trials was considered to be salient for that child. Following the cue salience pretest, one two-choice learning task, using the same color, form, shape, and position cues, was administered. Verbal feedback ("correct" or "incorrect") was given after each trial. The cue reinforced in the learning task was: (1) identical to the cue found to be salient in the pretest (SAME), (2) the alternate cue of the dimension represented by the pretest salient cue (REVERSE), (3) from one of the dimensions other than the pretest salient cue dimension (OTHER), or (4) determined randomly for those children who had responded inconsistently in the pretest, thus evidencing no specific salient cue (NO CUE). The learning task was presented until a criterion of 10 consecutive correct responses was attained or for 80 trials, whichever came first.

\section{RESULTS}

The children whose salient cue was relevant in the discrimination task were expected to learn fastest (SAME group). Next should be the children who were reinforced on the salient dimension, but for choosing the nonsalient cue (REVERSE group). This hypothesis assumes that cue salience and dimensional salience are related. The slowest learning should have been by the children whose most salient cue was totally irrelevant (OTHER group). Finally, the children who did not 
Table 1

Mean Trials to Criterion for Each Group

\begin{tabular}{ccccc}
\hline & \multicolumn{4}{c}{ Group } \\
\cline { 2 - 5 } & SAME & REVERSE & OTHER & NO CUE \\
\hline Mean & 26.1 & 33.5 & 48.7 & 29.7 \\
\hline
\end{tabular}

appear to have a specific salient cue were expected to learn at a rate between the OTHER group and the REVERSE group. This expectation was based on the assumption that having a relevant cue from a salient dimension should be facilitative and having the salient cue irrelevant should interfere with learning.

In the pretest, only 45 of the 111 children $(41 \%)$ responded consistently to a single cue. The selections of these children were distributed equally across six cues $\left[\chi^{2}(5)=1.80\right.$; no child selected a position cue $]$. The $41 \%$ with a salient cue was close to the $38 \%$ reported by Offenbach et al. (1973) for third-grade children. These data were consistent with Offenbach's earlier study and supported their contention that salience can be ascertained for specific cues as well as for dimensions.

As can be seen in Table 1, the obtained rank order of the group means was as predicted for the three groups who had a salient cue. The SAME children learned fastest, followed by the REVERSE group, and then the OTHER group. The differences between trial means was significant only for the SAME vs. OTHER comparison $[t(28)=2.04, p<.05$, one-tailed $]$. The stability of the rank order of the three means was tested using a procedure described by Jonckheer (1954). Based on that test, the probability of the obtained rank order of the three means occurring by chance was .021 . These findings clearly support the conclusion that cue salience, as it was determined in the pretest, was related to acquisition of a discrimination learning task.

The children without a salient cue were expected to take longer to learn than the children trained with the cue or dimension relevant, but that did not happen. The NO CUE group mean was about halfway between the SAME and REVERSE groups, and it was not different from either [SAME, $\mathrm{t}(79)=.45$; REVERSE, $\mathrm{t}(28)=.46$; both ps $>.05$ ]. The NO CUE group, however, was significantly different from the OTHER group $[\mathrm{t}(79)=2.26, \mathrm{p}<.05]$.

\section{DISCUSSION}

The results of this study have several interesting implications. First, it was satisfying to find out that specific cue salience, as opposed to dimensional salience, was related to discrimination learning in a logical manner. Second, the data supported the possibility of making a distinction between specific cue salience and dimensional salience. Further investigation using a cue salience pretest rather than a dimensional salience pretest might result in a more precise definition of the relation between attentional processes and learning.

Another result was that the percentage of children who responded consistently to a specific cue was consistent with other studies (Offenbach et al., 1973; Reiber, 1969), but it was much lower than reported in traditional dimensional salience tasks. Perhaps children enter a learning situation with strong specific cue saliences rather than strong dimensional saliences, or even with no salient dimension at all (the latter children may attend more or less equally to all of the cues). The data indicated that the latter children's learning was not at all impaired by the absence of a salient cue. In other words, learning was significantly retarded when and only when there was present a specific cue that was particularly salient to the subject, and when that cue, or the dimension of that cue, was not relevant to the solution. Thus, there may be no facilitative effect from having a salient cue present and relevant during learning.

\section{REFERENCES}

Cole, M. A probe trial procedure for the study of children's discrimination learning and transfer. Journal of Experimental Child Psychology, 1976, 22, 499-510.

Esposito, N. J. Review of discrimination shift learning in young children. Psychological Bulletin, 1975, 82, 432-455.

JonCKHEER, A. R. A distribution-free K-sample test against ordered alternatives. Biometrika, 1954, 41, 133-145.

Offenbach, S. I., Baecher, R., \& White, M. Effect of method, instructions, and age on stimulus "preference" assessments. Journal of Genetic Psychology, 1973, 122, 253-261.

REIBER, M. Hypothesis testing in children as a function of age. Developmental Psychology, 1969, 1, 389-395.

Tighe, L. S., Tighe, T. J., Waterhouse, M. D., \& Vasta, R. Dimensional preference and discrimination shift learning in children. Child Development, 1970, 41, 737-746.

(Received for publication May 23, 1979.) 